\title{
Nouvelles données et aperçu de la statistique médicale 2013
}

La FMH s'engage depuis de nombreuses années pour des statistiques fiables et fondées, raison pour laquelle la division Données, démographie et qualité (DDQ) publie tous les ans les derniers faits et chiffres concernant la statistique médicale. Vous trouverez de plus amples informations sur la statistique médicale de la FMH et sur la statistique des membres ainsi que les principaux indicateurs sur le site internet de la FMH (www.fmh.ch $\rightarrow$ Services $\rightarrow$ Statistique $\rightarrow$ Statistique médicale).

Stefanie Hostettler ${ }^{a}$, Esther Kraft ${ }^{b}$

a Dr sc. EPFZ, division Données, démographie et qualité (DDQ) de la FMH

b lic. rer. oec., cheffe de la division Données, démographie et qualité (DDQ) de la FMH
Correspondance:

FMH/division DDQ

Elfenstrasse 18

CH-3000 Berne 15

Tél. 0313591111

ddq[at]fmh.ch

\section{Introduction}

Les conditions-cadres et les exigences du système de santé suisse sont en perpétuelle mutation. Or pour que le système de santé puisse continuer à fonctionner à un niveau de qualité élevé, il importe de montrer les évolutions et les tendances et de prendre les mesures et corrections qui s'imposent. Dans ce contexte, la statistique médicale de la FMH représente une source de données essentielle pour élaborer des bases et des indicateurs en matière de recherche démographique et de recherche sur les soins

La statistique médicale est établie à la fin de chaque année (jour de référence: 31 décembre) et décrit notamment les principales caractéristiques de la démographie médicale telles que l'âge, le sexe, la nationalité et le lieu où le médecin exerce sa profession. La banque de données de la FMH, qui comporte les données socio-démographiques de plus de

Figure 1

Vue générale des médecins en exercice de 1960 à 2013.

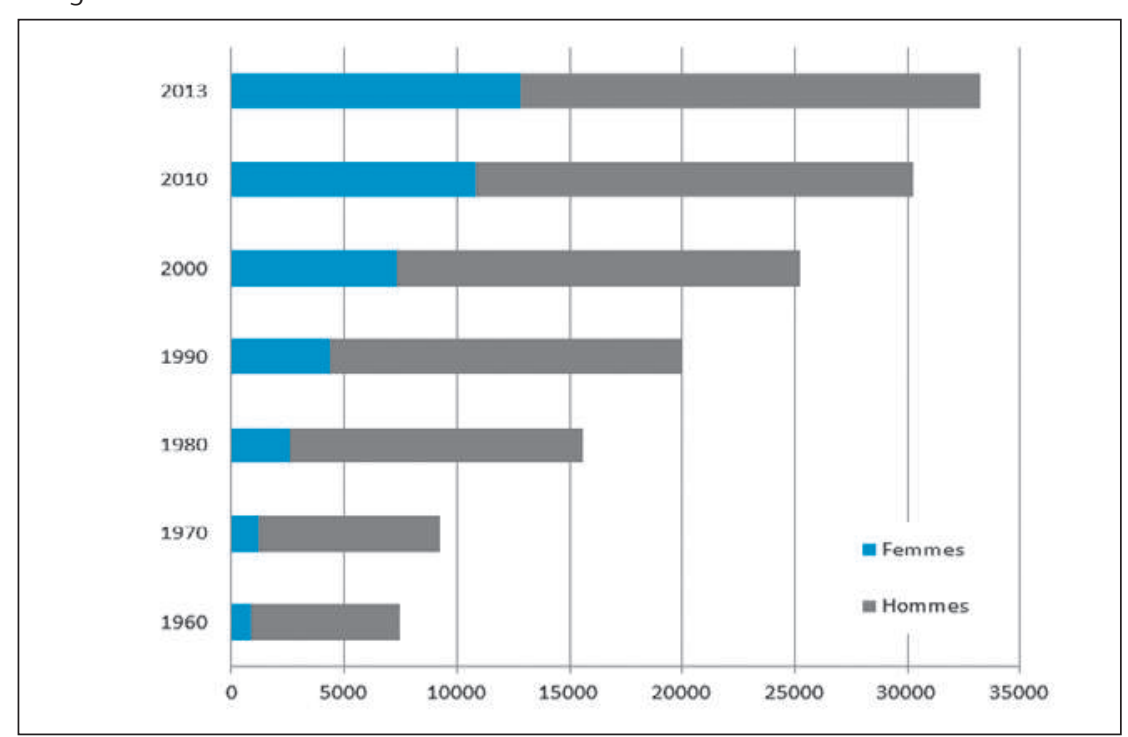

33000 médecins sert de base à cette analyse. Le premier volet de la statistique de la FMH est consacré aux spécificités démographiques des médecins en exercice ainsi qu'à leur activité professionnelle principale et le second se penche sur les données autodéclarées par plus de 10000 médecins au moyen d'un questionnaire sur le portail des membres myFMH.

Le présent article propose un choix thématique. Les informations détaillées, les tableaux, graphiques, dépliants et statistiques médicales des années précédentes ainsi que les indicateurs sont disponibles sur le site internet de la FMH (www.fmh.ch $\rightarrow$ Services $\rightarrow$ Statistique $\rightarrow$ Statistique médicale). Y figure également un outil de recherche interactif qui permet de trouver rapidement les informations souhaitées, d'éditer des tableaux ou des graphiques, de les enregistrer et de les imprimer.

\section{Augmentation du nombre de médecins}

En 2013, 33242 médecins exerçaient en Suisse, ce qui correspond à une augmentation de 4,3\% par rapport à l'année précédente. La part de femmes dans la profession connaît une augmentation constante et atteint actuellement 38,6\% (12816 femmes, 20426 hommes). L'augmentation est plus importante chez les femmes (7,1\%) que chez les hommes $(2,7 \%)$, ce qui reflète la progression du nombre d'étudiantes en médecine humaine au cours de ces dernières années. A l'instar des années précédentes, davantage de femmes que d'hommes ont obtenu leur diplôme de médecin (sur 541 masters, 56,4\% ont été obtenus par des femmes et sur 646 doctorats, $53,1 \%$ ont été obtenus par des femmes) [1].

La division DDQ se tient volontiers à votre disposition pour tout complément d'information: ddq[at]fmh.ch / 0313591111. 
* Les données relatives à la densité médicale se rapportent à l'année 2012, étant donné que les effectifs de la population recensés par l'OFS n'étaient pas encore disponibles au moment de la publication de la statistique médicale.

\section{Plus de la moitié des médecins exercent dans le secteur ambulatoire}

$52,8 \%$ des médecins exercent leur activité principale dans le secteur ambulatoire, alors que 45,5\% exercent dans le secteur hospitalier et 1,7\% dans un autre secteur (assurances, associations). La part des femmes est plus élevée dans le secteur hospitalier (43,3\%) que dans le secteur ambulatoire (34,8\%). En Suisse, les médecins exercent principalement dans un seul secteur $(89,5 \%)$, mais une petite partie d'entre eux pratique dans plus d'un secteur (10,5\%). Parmi ceuxci, la majorité $(84,1 \%)$ exerce son activité principale dans le secteur ambulatoire tout en occupant simultanément un autre poste dans le domaine hospitalier, notamment en tant que médecins agréés.

La tendance est la même lorsqu'on considère le nombre de lieux de travail. La majorité des médecins $(86,4 \%)$ exerce sur un seul site, tandis que $11,1 \%$ par-

Figure 2

Médecins en exercice par secteur (activité principale) en 2013.

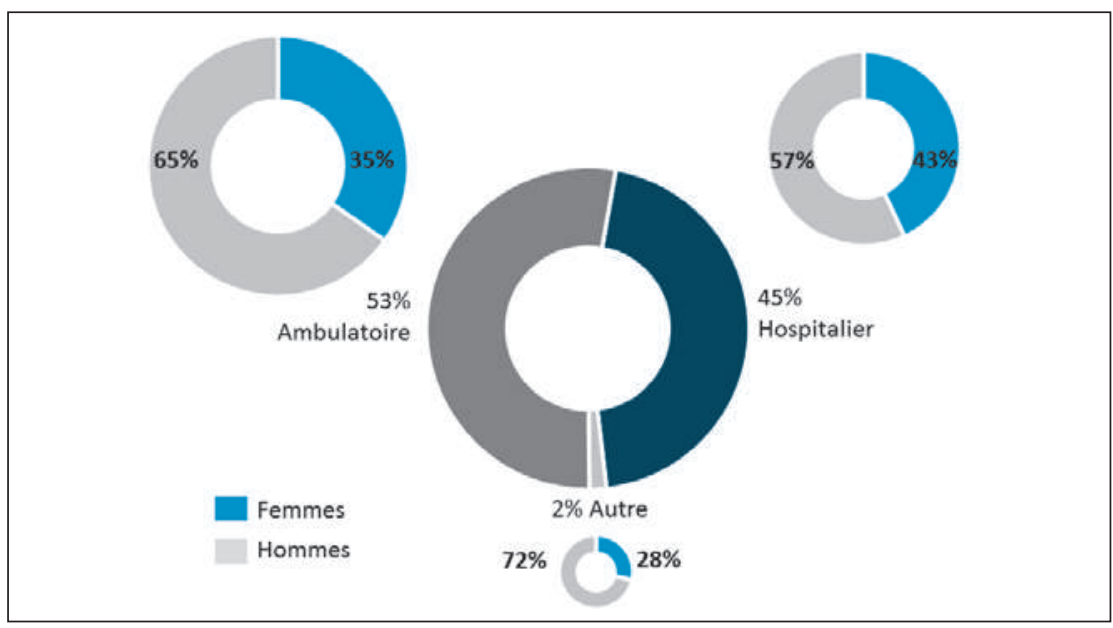

\section{Tableau 1}

Aperçu de l'activité des médecins par secteur (activité principale) en 2013.

\begin{tabular}{llll} 
& Femmes & Hommes & Total \\
\hline Secteur ambulatoire & 6109 & 11445 & 17554 \\
\hline Secteur hospitalier & 6548 & 8579 & 15127 \\
\hline Autre secteur & 159 & 402 & 561 \\
\hline Total & 12816 & 20426 & 33242
\end{tabular}

Figure 3

Densité médicale par canton en 2012 (nombre de médecins par 1000 habitants).

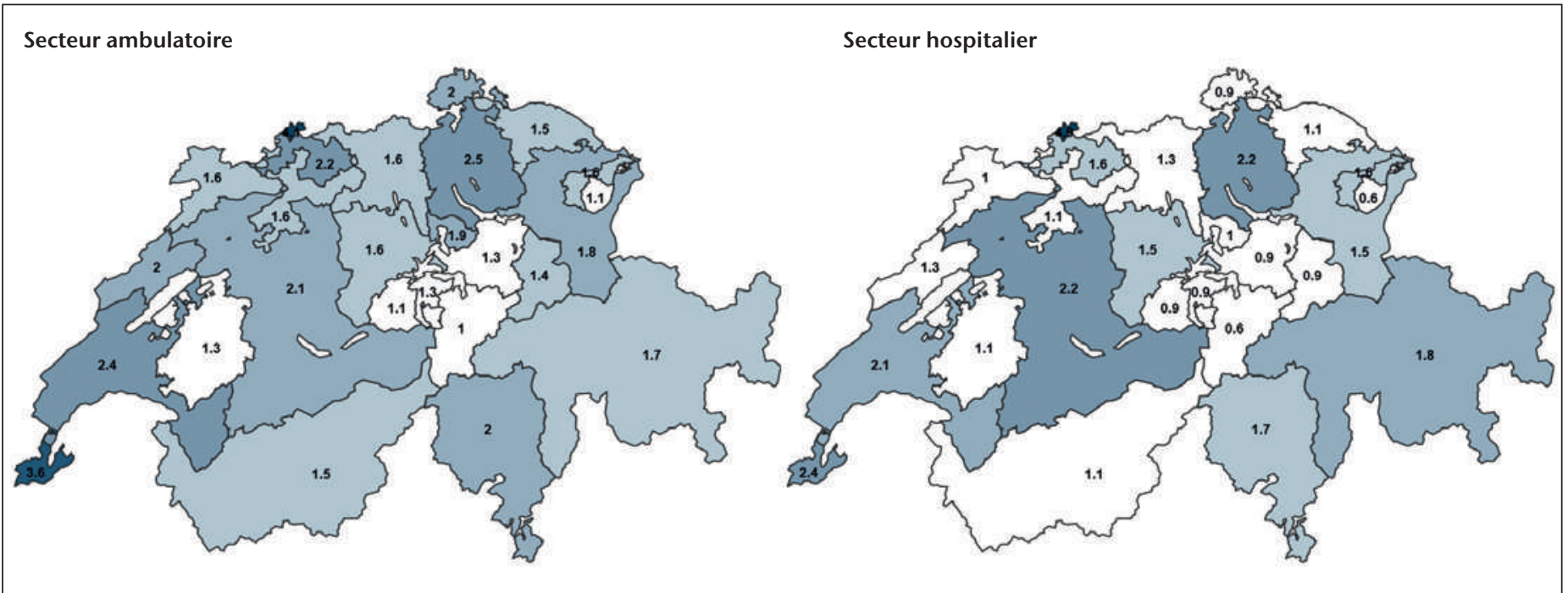




\section{La médecine interne générale est la discipline la plus courante}

La médecine interne générale $(27,8 \%)$, la psychiatrie et psychothérapie $(12,1 \%)$ et la gynécologie et obstétrique $(5,6 \%)$ sont les disciplines les plus représen-

\section{Tableau 2}

Aperçu du nombre de médecins par discipline (activité principale) en 2013.

\begin{tabular}{|c|c|c|c|}
\hline & Femmes & Hommes & Total \\
\hline Allergologie / Immunologie & 52 & 99 & 151 \\
\hline Anesthésiologie & 558 & 797 & 1355 \\
\hline Angiologie & 41 & 131 & 172 \\
\hline Cardiologie & 93 & 557 & 650 \\
\hline Chir. cardiaque et vasc. thor. & 5 & 78 & 83 \\
\hline Chirurgie & 177 & 931 & 1108 \\
\hline Chirurgie de la main & 37 & 133 & 170 \\
\hline Chirurgie orale et maxillo-faciale & 7 & 80 & 87 \\
\hline Chirurgie orthopédique & 73 & 874 & 947 \\
\hline Chirurgie pédiatrique & 24 & 50 & 74 \\
\hline Chirurgie plastique & 43 & 129 & 172 \\
\hline Dermatologie et vénéréologie & 229 & 268 & 497 \\
\hline Endocrinologie / Diabétologie & 70 & 113 & 183 \\
\hline Gastroentérologie & 34 & 292 & 326 \\
\hline Génétique médicale & 14 & 10 & 24 \\
\hline Gynécologie et obstétrique & 880 & 722 & 1602 \\
\hline Hématologie & 48 & 112 & 160 \\
\hline Infectiologie & 49 & 103 & 152 \\
\hline Méd. phys. et réadaptation & 57 & 128 & 185 \\
\hline Médecin praticien & 587 & 629 & 1216 \\
\hline Médecine du travail & 37 & 69 & 106 \\
\hline Médecine intensive & 153 & 358 & 511 \\
\hline Médecine interne générale & 2636 & 5244 & 7880 \\
\hline Médecine légale & 18 & 32 & 50 \\
\hline Médecine nucléaire & 12 & 55 & 67 \\
\hline Médecine pharmaceutique & 20 & 37 & 57 \\
\hline Médecine tropicale et des voyages & 8 & 22 & 30 \\
\hline Néphrologie & 60 & 119 & 179 \\
\hline Neurochirurgie & 21 & 139 & 160 \\
\hline Neurologie & 163 & 367 & 530 \\
\hline Neuropathologie & 3 & 13 & 16 \\
\hline Oncologie médicale & 101 & 192 & 293 \\
\hline Ophtalmologie & 369 & 566 & 935 \\
\hline ORL & 110 & 343 & 453 \\
\hline Pathologie & 108 & 122 & 230 \\
\hline Pédiatrie & 866 & 693 & 1559 \\
\hline $\begin{array}{l}\text { Pharmacologie et toxicologie } \\
\text { clinique }\end{array}$ & 17 & 25 & 42 \\
\hline Pneumologie & 48 & 217 & 265 \\
\hline Prévention et santé publique & 36 & 43 & 79 \\
\hline $\begin{array}{l}\text { Psychiatrie d'enfants et } \\
\text { d'adolescents }\end{array}$ & 377 & 226 & 603 \\
\hline Psychiatrie et psychothérapie & 1427 & 1999 & 3426 \\
\hline Radiologie & 204 & 564 & 768 \\
\hline Radio-oncologie / Radiothérapie & 40 & 66 & 106 \\
\hline Rhumatologie & 106 & 330 & 436 \\
\hline Urologie & 27 & 262 & 289 \\
\hline
\end{tabular}

tées. Dans le secteur ambulatoire, la répartition est la même: les généralistes forment le groupe professionnel le plus important (médecine interne générale $34,3 \%)$, suivi par les psychiatres (14,4\%) et les gynécologues $(6,3 \%)$. Dans le secteur hospitalier, la médecine interne générale est également en tête (17,7\%), suivie par l'anesthésiologie $(9,7 \%)$, la psychiatrie et la psychothérapie $(8,4 \%)$.

On trouve le plus grand nombre de femmes médecins en psychiatrie et psychothérapie d'enfants et d'adolescents $(62,4 \%)$, en pédiatrie $(55,5 \%)$ et en gynécologie et obstétrique (54,9\%). Comparés aux femmes, les hommes sont nettement surreprésentés dans les disciplines chirurgicales (chirurgie cardiaque et vasculaire thoracique $(94,0 \%)$, chirurgie orthopédique $(92,3 \%)$, neurochirurgie $(86,9 \%)$, et chirurgie $(84,0 \%))$.

\section{Pyramide des âges du corps médical}

En Suisse, la moyenne d'âge des médecins se situe à 48,8 ans (cf. tabl. 3). Un médecin du secteur ambulatoire a en moyenne 53,7 ans, tandis que son collègue du secteur hospitalier a 43,0 ans. Cette différence d'âge entre les secteurs ambulatoire et hospitalier s'explique par le fait que la formation postgraduée des assistants se déroule essentiellement en milieu hospitalier. Au moment de l'obtention de leur titre de spécialiste, autrement dit au terme de leur formation postgraduée, les médecins ont 37 ans en moyenne.

La pyramide des âges de la figure 4 illustre la répartition sexo-spécifique du corps médical en fonction de l'âge. En dessous de 35 ans, le nombre de femmes est majoritaire dans la profession. A partir de 40 ans, le nombre d'hommes en exercice dépasse actuellement celui des femmes. Mais la hausse de la proportion de femmes chez les étudiants et chez les jeunes médecins devrait se refléter dans les différentes tranches d'âge au cours des prochaines années.

\section{Répartition des médecins en exercice selon leur fonction}

Parmi les 17554 médecins en exercice dans le secteur ambulatoire, $84,3 \%$ sont propriétaires ou propriétaires associés de leur cabinet médical. 3,5\% des médecins sont engagés en tant qu'assistant ou spécialiste au cabinet médical. Le reste des médecins de ce secteur exerce une autre fonction. Les médecinsassistants en formation postgraduée (50\%) représentent le groupe le plus important du secteur hospi-

\section{Tableau 3}

Age moyen du corps médical en 2013.

\begin{tabular}{llll} 
& Femmes & Hommes & Tous \\
\hline Secteur ambulatoire & 50,6 & 55,3 & 53,7 \\
\hline Secteur hospitalier & 39,7 & 45,5 & 43,0 \\
\hline Tous les secteurs & 45,0 & 51,2 & 48,8
\end{tabular}


Figure 4

Pyramide des âges et répartition sexo-spécifique des médecins en exercice en 2013.

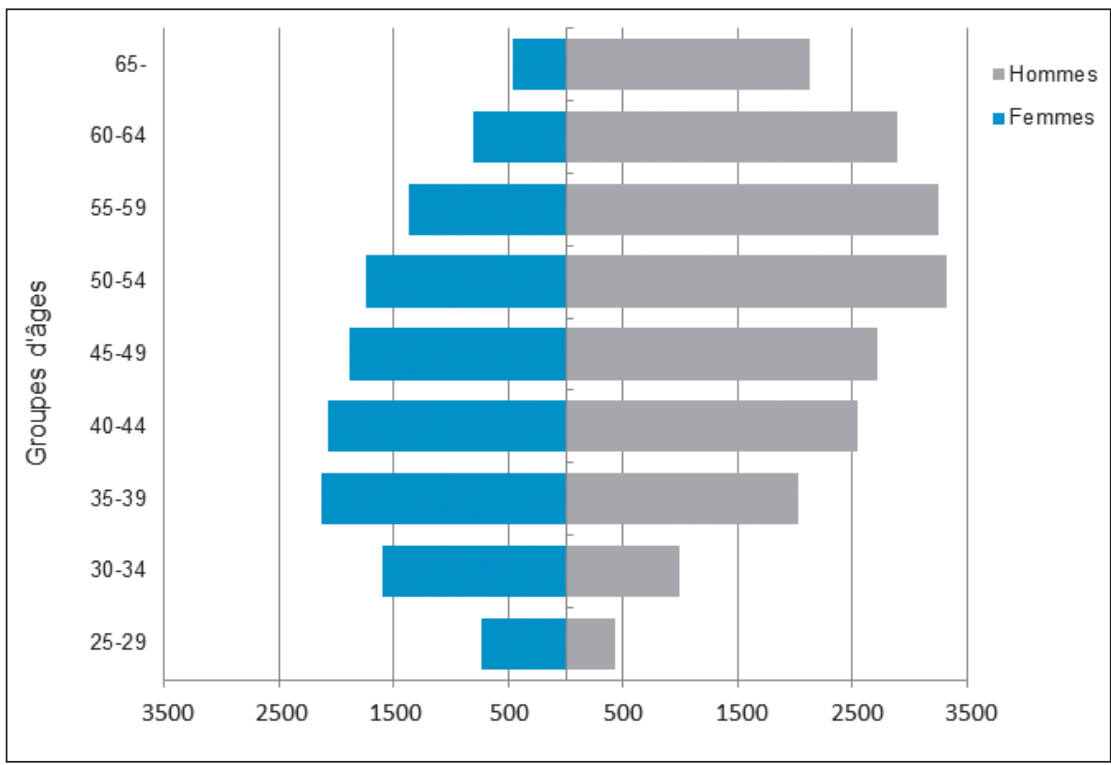

Figure 5

Nombre de médecins avec un diplôme étranger en 2013.

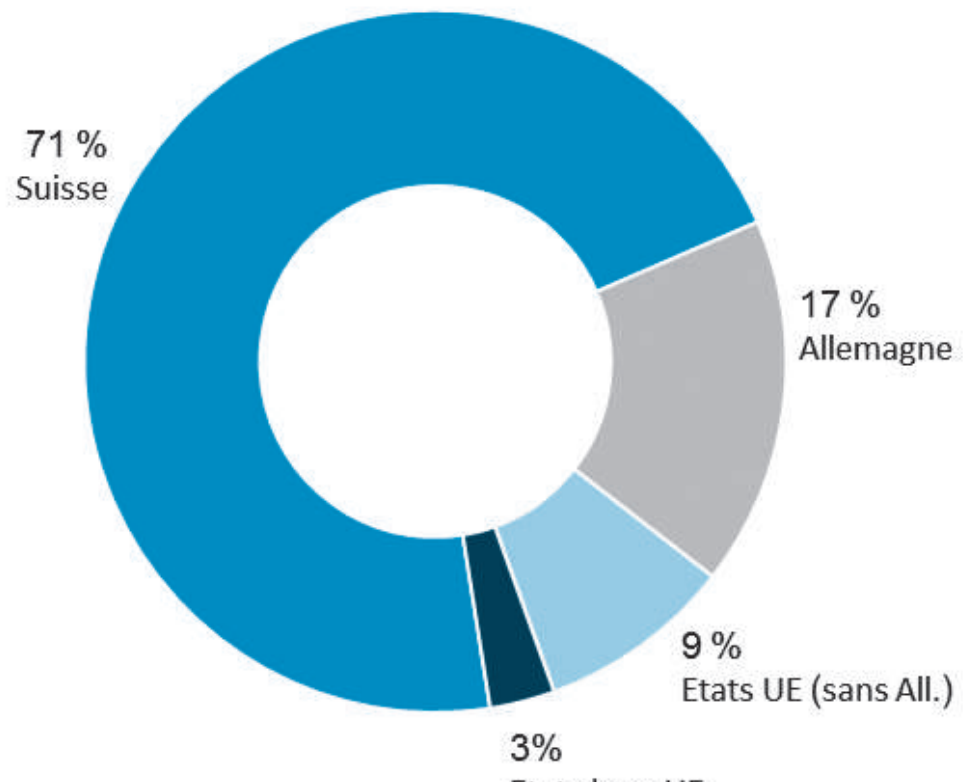

Etats hors UE

Tableau 4

Taux d'occupation moyen par secteur et par sexe en demijournées par semaine en $2013(\mathrm{~N}=6432)$.

\begin{tabular}{llll} 
& Femmes & Hommes & Total \\
\cline { 2 - 4 } Secteur ambulatoire & 6,8 & 8,8 & 8,2 \\
\hline Secteur hospitalier & 8,8 & 10,1 & 9,5 \\
\hline Autre secteur & 7,8 & 9,0 & 8,6 \\
Total & 7,4 & 9,2 & 9,0
\end{tabular}

talier. Comme on peut s'y attendre, le nombre de médecins dans ce dernier groupe tend à diminuer plus le degré hiérarchique est élevé. On compte par ex. 20,1\% de chefs de clinique, $12,4 \%$ de médecins adjoints et $10,2 \%$ de médecins-chefs. Le nombre de femmes prédomine uniquement chez les médecinsassistants $(56,7 \%)$ et ne fait que diminuer ensuite pour atteindre $42,1 \%$ chez les chefs de clinique, $22,4 \%$ chez les médecins adjoints et seulement $10,3 \%$ chez les médecins-chefs.

\section{Médecins étrangers}

En Suisse, 9756 médecins sont détenteurs d'un diplôme de médecin étranger (fig. 5). Parmi ces derniers, 56,3\% exercent dans le domaine hospitalier, $41,5 \%$ dans le secteur ambulatoire et 2,2\% dans un autre domaine. La majorité des médecins étrangers vient d'Allemagne $(16,9 \%)$ ou des autres pays de l'Union européenne $(9,1 \%)$. En comparaison avec l'année précédente, le nombre de médecins en exercice au bénéfice d'un diplôme étranger a progressé de $1,8 \%$ pour atteindre $29,4 \%$.

Parmi les 1433 titres de spécialiste décernés en 2013, 37\% l'ont été à des médecins au bénéfice d'un diplôme de médecin de l'un des pays suivants: Allemagne $68,6 \%$, Autriche $10,2 \%$, Italie $6,4 \%$, autres $14,8 \%$ (source: ISFM).

\section{Taux d'occupation plus élevé dans le domaine hospitalier}

Les indications concernant le taux d'occupation et toutes les évaluations figurant dans ce second volet se fondent sur les données déclarées par les médecins au moyen d'un questionnaire sur le portail des membres myFMH. En raison d'un biais de sélection, les facteurs que sont le secteur, le sexe et la discipline ont été analysés et comparés avec la population-mère. Là où l'écart entre l'échantillon et la population-mère dépassait 10\% (nombre de femmes dans le secteur ambulatoire), une pondération a été effectuée.

Les indications concernant le taux d'occupation ont été validées dans le cadre d'une étude menée conjointement avec NewIndex [3]. Cette dernière a révélé que les données de la FMH fournies par autodéclaration se recoupaient bien avec les données de facturation des prestations, offrant ainsi des indications fiables et représentatives.

En 2013, les médecins ont travaillé en moyenne 9,0 demi-journées (cf. tabl. 4) par semaine. Dans le secteur ambulatoire, le taux d'activité moyen était de 8,2 demi-journées, à savoir 1,5 demi-journée de moins que dans le secteur hospitalier (9,5 demi-journées). Le taux d'occupation moyen des femmes médecins (en ambulatoire: 6,8; en hospitalier: 8,8 demi-journées) est inférieur à celui de leurs collègues masculins (en ambulatoire: 8,8; en hospitalier: 10,1 demi-journées). Ce modèle n'a quasiment pas changé au cours des cinq dernières années. 


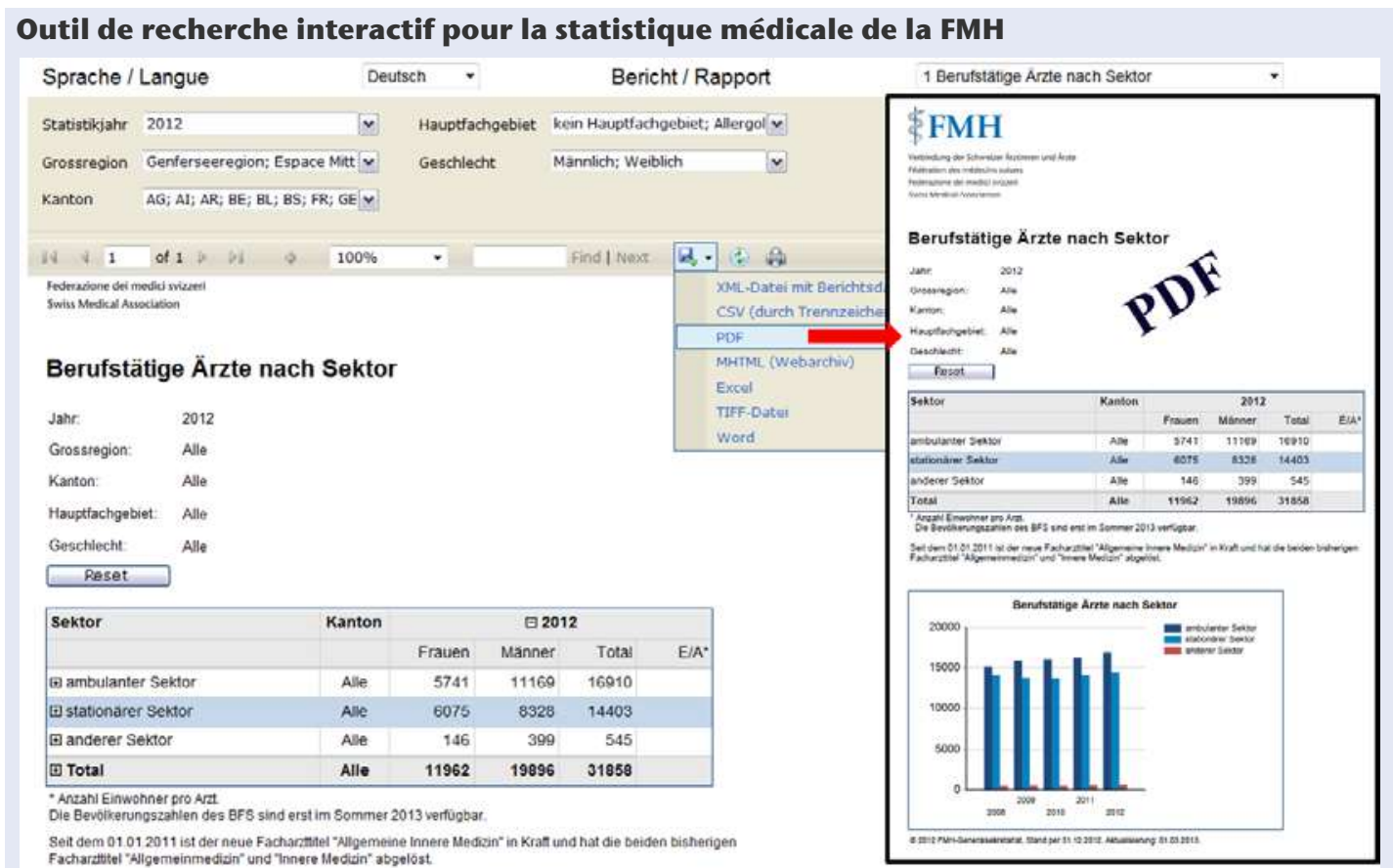

Le site de la statistique médicale www.fmh.ch $\rightarrow$ Services $\rightarrow$ Statistiques $\rightarrow$ Statistique médicale propose un outil interactif pour trouver soi-même les informations recherchées sur la statistique médicale. La recherche peut ensuite être imprimée ou enregistrée et exportée dans différents formats (.pdf, .xls, .csv, etc.). En cas de question concernant l'utilisation de l'interface et pour toute autre question, la division Données, démographie et qualité (DDQ) se tient à votre entière disposition (ddq[at]fmh.ch / 03135911 11).

Alors que dans le domaine hospitalier, la majorité des médecins $(80,0 \%)$ travaille à plein temps, ce taux est nettement inférieur dans le domaine ambulatoire $(56,6 \%)$. La figure 6 montre que cet écart entre les deux secteurs est principalement sexo-spécifique. Si dans le domaine ambulatoire, 31,0\% des hommes travaillent à temps partiel, ce taux atteint $73,2 \%$ chez les femmes. Les femmes médecins privilégient un taux d'occupation de $60 \%$ à $80 \%$. Dans le domaine hospitalier, la majorité des femmes $(59,7 \%)$ et des hommes $(87,7 \%)$ travaille à plein temps. Cela est probablement dû au fait que la formation postgraduée des médecins a principalement lieu en milieu hospitalier et que pendant cette période professionnelle, il est plus rare de réduire son temps de travail.

Figure 6

Taux d'occupation par sexe et par secteur en 2013.
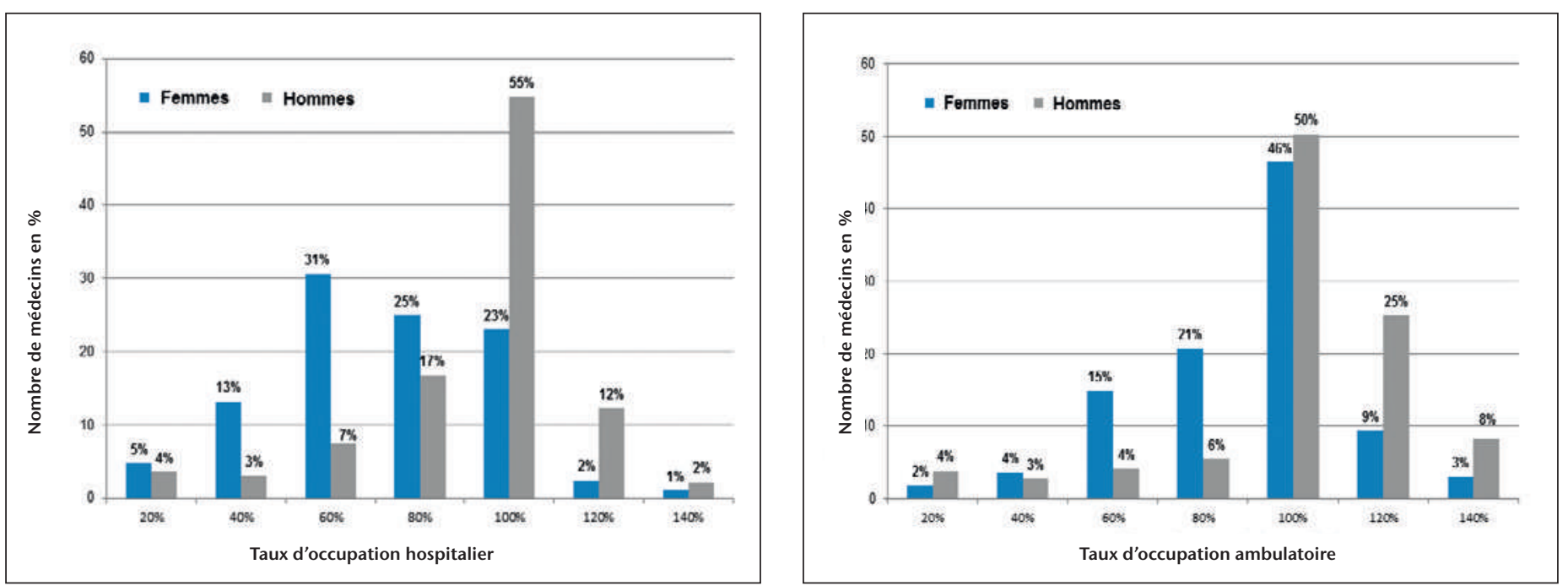
Structures de cabinets et réseaux de médecins

Dans le secteur ambulatoire, les médecins exercent principalement en cabinet individuel $(58,6 \%)$. Depuis 2008 , ce taux a diminué de $5 \%$ environ. Les raisons de cette diminution résident probablement dans le souhait de travailler en équipe ou à temps partiel, ainsi que dans un rapport de salarié.

Comparés aux femmes (49,7\%), les hommes médecins exercent plus souvent en cabinet individuel $(62,9 \%)$. Le nombre de femmes exerçant en cabinet de groupe ou en cabinets à deux médecins est plus élevé (50,3\%) que chez les hommes (37,1\%).

Selon les données auto-déclarées, 48,5\% des médecins exerçant dans le domaine ambulatoire sont affiliés à un réseau de médecins. Alors que $50,6 \%$ d'hommes médecins sont dans un réseau, la part de leurs collègues féminins est légèrement inférieure et atteint $43,7 \%$. Le nombre total de médecins affiliés à un réseau reste toutefois identique à celui de l'année précédente.

\section{Conclusions}

Les résultats de la statistique médicale 2013 fournissent un aperçu différencié du corps médical en Suisse et forment une base de données fondée sur des preuves en vue de prises de décisions politiques. Sur la base de ces données exhaustives et fiables, la FMH vise d'autres améliorations (par ex. la subdivision du secteur ambulatoire en ambulatoire hospitalier et ambulatoire au cabinet). C'est également dans ce sens et conformément au rapport «Santé 2020» [4] que les priorités de ces prochaines années en politique de la santé doivent avoir pour but d'améliorer la transparence, de combler les lacunes statistiques et de récolter des données fiables sur la qualité des prestations médicales. Grâce à la statistique médicale et à l'Académie suisse pour la qualité en médecine (ASQM), la FMH dispose d'outils appropriés pour réaliser et développer ces objectifs, ce qui ne serait cependant pas possible sans la collaboration du corps médical. La FMH saisit donc cette occasion pour remercier aux membres FMH pour leur engagement tout en espérant qu'elle pourra continuer à compter sur leur soutien à l'avenir.

\section{Grâce à vous, la statistique médicale de la FMH dispose de données de bonne qualité!}

Pour obtenir des données de bonne qualité, la FMH a besoin du soutien de tous ces membres. Quelques minutes sont nécessaires pour remplir et vérifier le questionnaire sur l'activité professionnelle mais l'impact pour l'ensemble du corps médical est très important. Enregistrez-vous sur le portail internet des membres myFMH. Vous trouverez le questionnaire sur la page d'accueil. Nous sommes à votre disposition pour tout complément d'information par courriel à myfmh[at]fmh.ch ou par téléphone au 031 3591259.

La FMH tient à remercier tous les médecins ayant déclaré ou vérifié leurs données sur myFMH pour leur précieuse collaboration. Grâce à eux, nous disposons de statistiques médicales fiables.

\section{Références}

1 www.bfs.admin.ch/bfs/portal/fr/index/themen/15/06/ data/blank/02.html

2 OCDE (2013), Panorama de la santé 2013: Les indicateurs de l'OCDE, Éditions OCDE. http://dx.doi. org/10.1787/health_glance-2013-fr

3 Hostettler S, Laffranchi R, Kraft E. Taux d'occupation des médecins en Suisse. Bull Méd Suisses. 2013;95(50):1891-5

4 www.bag.admin.ch/gesundheit2020/index. html?lang=fr

\section{Articles interactifs}

Vous souhaitez commenter cet article? II vous suffit pour cela d'utiliser la fonction «Ajouter un commentaire» dans la version en ligne. Vous pouvez également consulter les remarques de vos confrères sous: www.bullmed.ch/ numero-actuel/articles-interactifs/ 


\section{Glossaire (par ordre alphabétique)}

Activité principale

Est considéré comme activité principale le secteur dans lequel le médecin exerce la majeure partie de son activité.

\section{Autre secteur}

Font partie d'un autre secteur les activités de médecins ne relevant ni du secteur ambulatoire ni du secteur hospitalier, par ex. professeurs, privat-docents, médecins engagés par des assurances, etc.

Cabinet à deux médecins ou de groupe Utilisation d'appareils, d'équipements, de locaux ou de personnel par deux ou plusieurs médecins.

\section{Cabinet individuel}

Utilisation d'appareils, d'équipements, de locaux ou de personnel par un seul médecin.

\section{Canton}

Le canton pris en compte est celui dans lequel le médecin exerce son activité principale. S'il n'a rien indiqué à ce sujet, c'est le canton de l'adresse de contact qui est pris en compte.

\section{Discipline principale}

La discipline principale d'un médecin correspond au titre de spécialiste dans le cadre duquel il exerce la majeure partie de son activité médicale (d'après son autodéclaration et les règles prédéfinies).

\section{Médecins du secteur ambulatoire}

Médecins qui exercent leur activité principale dans le secteur ambulatoire.

\section{Médecins du secteur hospitalier}

Médecins qui exercent leur activité principale en milieu hospitalier.

Médecins d'un autre secteur

Médecins qui n'exercent leur activité principale ni dans le secteur ambulatoire ni en milieu hospitalier.

\section{Réseaux de médecins}

Les réseaux de médecins sont des organisations formées par des prestataires de soins et assurant la couverture médicale. Sur la base d'une collaboration contractuelle tant mutuelle qu'avec des fournisseurs et des organismes extérieurs au réseau, ils fournissent des prestations de santé axées sur les besoins des patients. Cette collaboration repose sur un processus thérapeutique convenu par contrat, des structures organisationnelles entrepreneuriales et une culture commune de la prise en charge des patients.

\section{Secteur ambulatoire}

Font partie du secteur ambulatoire les consultations données et les soins dispensés par des médecins en cabinet individuel ou de groupe. Les patients sont généralement traités de manière ambulatoire ou à leur domicile (visites à domicile). Font également partie du secteur ambulatoire les activités des médecins consultants privés dans les hôpitaux ou dans des dispensaires, des infirmeries ou des établissements analogues attachés à des entreprises, des écoles, des homes pour personnes âgées, des organisations syndicales et des confréries (cabinet médical indépendant ou semblable). (Source: OFS)

\section{Secteur hospitalier}

Font partie du secteur hospitalier les traitements médicaux, diagnostics, soins, interventions chirurgicales, analyses, services d'urgence et activités de formation prégraduée, postgraduée et continue, etc. dans les hôpitaux. Font également partie du secteur hospitalier les foyers protégés avec un encadrement social $24 \mathrm{~h}$ sur 24 accueillant des enfants, des personnes âgées et des groupes de personnes dont l'autonomie est limitée. (Source: OFS)

\section{Taux d'occupation}

Le taux d'occupation est indiqué en demi-jours.

Un demi-jour correspond à un volume de travail de 4 à 6 heures. Un emploi à plein temps correspond à un taux d'occupation hebdomadaire moyen de 10 demi-jours.

Traitements ambulatoires en milieu hospitalier Tous les traitements qui ne sont pas réputés hospitaliers ou semi-hospitaliers.

Traitements hospitaliers en milieu hospitalier Séjours à l'hôpital d'au moins 24 heures pour des examens, des traitements et des soins. Séjours à l'hôpital de moins de 24 heures, au cours desquels un lit est occupé durant une nuit. Séjours à l'hôpital en cas de transfert dans un autre hôpital ou en cas de décès. 